\title{
Intereses de formación, motivación y sentido de vida de trabajadores en proceso de prejubilación
}

\section{Sara Esperanza Lucero Revelo}

orcid.org/0000-0003-2232-2863

Universidad Mariana, Colombia

slucero@umariana.edu.co

\section{Eunice Yarce Pinzón}

orcid.org/0000-0003-4084-1296 Universidad Mariana, Colombia eyarce@umariana.edu.co

\section{Resumen}

Se determinaron los intereses de formación, motivación y sentido de vida de los trabajadores en proceso de prejubilación, con el fin de proponer estrategias educativas pertinentes. Para hacer esto, se tuvo como base el paradigma cuantitativo, con un muestreo no probabilístico por conveniencia. La muestra fue tomada de un grupo de trabajadores de una universidad privada colombiana, lo que circunscribe la validez de las conclusiones a contextos similares. Los resultados muestran que el mayor interés de los trabajadores en proceso de prejubilación, para formación permanente, están dirigidos a temas de salud, informática, relaciones humanas y emprendimiento.

\section{Palabras clave}

Motivación; formación permanente; educación continuada; sentidos de vida; jubilación (Fuente: Tesauro de la Unesco).

Recepción: 08/05/2017 | Envío a pares: 30/10/2017 | Aceptación por pares: 15/11/2017 | Aprobación:30/11/2017 


\title{
Interests of Training, Motivation and Sense of Life in Workers in the Process of Early Retirement
}

\begin{abstract}
Interests with respect to training, motivation and sense of life among workers who are in the process of early retirement were determined in order to propose pertinent educational strategies. The quantitative paradigm used to accomplish this was based on non-probabilistic convenience sampling. The sample was taken from a group of workers at a private university in Colombia, which circumscribes the validity of the conclusions to similar contexts. The results show the greatest interest among workers in an early retirement process, with respect to ongoing training, are focused on health, information technology, human relations and issues related to entrepreneurship.
\end{abstract}

Keywords

Motivation; lifelong education; retirement; Colombia (Source: Unesco Thesaurus). 


\section{Interesses de formação, motivação e sentido de vida de trabalhadores em processo de pré-aposentadoria}

Resumo

Neste estudo, determinaram-se os interesses de formação, motivação e sentido de vida dos trabalhadores em processo de pré-aposentadoria, a fim de propor estratégias educativas pertinentes. Para isso, teve-se como base o paradigma quantitativo com uma amostra não probabilística por conveniência. Esta foi tomada de um grupo de trabalhadores de uma universidade particular colombiana, o que circunscreve a validade das conclusões a contextos semelhantes. Os resultados mostram que o maior interesse dos trabalhadores em processo de pré-aposentadoria, para formação permanente, está dirigido a temas de saúde, informática, relações humanas e empreendimento.

\section{Palavras-chave}

Motivação; educação contínua; sentidos de vida; aposentadoria (Fonte: Tesauro da Unesco). 
ISSN 0123-1294 | e-ISSN 2027-5358 | Educ.Educ. Vol. 21. No.1 | Enero-abril de 2018 | pp. 95-113.

Universidad de La Sabana | Facultad de Educación

\section{Introducción}

Partiendo de la claridad que ofrece el concepto de envejecimiento con éxito, propuesto por Rowe y Kahn (1987) en la década de los ochenta, para evitar que se lo considere como proceso equivalente al de enfermedad y tratar de ofrecer una perspectiva más optimista de las últimas décadas de la vida, envejecer satisfactoriamente implica tres elementos: salud física, con baja probabilidad de padecer enfermedades y discapacidades asociadas; capacidad funcional alta, física y cognitiva, que permita realizar las actividades de la vida diaria, y compromiso activo con la vida. En consecuencia, envejecer satisfactoriamente significa estar libre de enfermedades graves, disponer de buen funcionamiento físico y mental, de tal manera que la persona realice sin dificultad y de manera autónoma sus actividades diarias, con la capacidad de implicarse activa y eficazmente en la vida con patrones de actividades determinados. De acuerdo con Rowe y Kahn (1998, citados por Villar, 2013), este patrón se relaciona con el mantenimiento de las relaciones interpersonales satisfactorias y con el mantenimiento de las actividades productivas.

Según López (2013), el surgimiento de programas universitarios dirigidos a personas mayores en América Latina que se planteó hace 25 años aproximadamente ha afrontado diversas dinámicas y retos, con profesionales basados en su experiencia, recursos personales y el aprendizaje rápido, resultado obligado de la interacción permanente con los estudiantes mayores, para dar respuesta a sus requerimientos, demandas y expectativas. Este autor diferencia los retos en académicos, curriculares, metodológicos, económicos y de gestión, que deben ser analizados y tenidos en cuenta para definir programas de educación permanente para la población de adultos mayores.

Por otro lado, López también plantea retos en la propia interacción con los estudiantes mayores, como la necesidad o pertinencia de un trato distinto, el conocimiento necesario para comprender esta etapa de la vida y las circunstancias que pueden es- tar viviendo, que implican determinadas conductas, el adecuado manejo de la disciplina y el respeto de las normas institucionales, combinados con flexibilidad y tolerancia, trato personalizado, tanto en temas institucionales como en personales, y adecuado manejo de sus expectativas educativas, entre otros.

La educación dirigida al adulto mayor, para que propicie el desarrollo de dichas capacidades, debe partir del principio de que el ser humano sigue en formación hasta el último día de su vida, y si no hay una edad específica para aprender, porque el individuo puede aprender a lo largo de toda su vida, entonces tampoco hay una sola edad para desarrollar un pensamiento crítico, reflexivo, cuestionador. Debe partirse de que los mayores pueden y deben seguir desarrollándose como seres humanos y que pueden y deben desarrollar nuevas habilidades. Por ello, no se trata de ayudarlos a adaptarse al sistema o a su realidad o de brindarles cursos y talleres para que se entretengan, sino de constituirlos en sujetos críticos, capaces de pensar y actuar sobre dicha realidad, para transformar su entorno con las acciones que puedan desarrollar en su familia, su comunidad, su ciudad o su país.

Es así, tal y como lo afirma Torres (2009), la educación debe estar inspirada en procesos que permitan el protagonismo no solo en la construcción del conocimiento y en el desarrollo de la crítica, sino en la intervención social. De allí que sea necesario, por un lado, promover en el aula el desarrollo de habilidades comunicativas y pensamientos autónomos, estructurados reflexivamente con disposición a la crítica y al diálogo, y por otro, replantear el rol del docente, conductor o guía del proceso de aprendizaje empoderante. Su papel está vinculado estrechamente a la definición de los objetivos, currículos, estrategias y metodologías, así como a las formas de transmitir el conocimiento y de construirlo conjuntamente con los educandos mayores, en un ambiente incluyente que respete y recoja las diferencias y la libertad de ideas, pero en un marco de respeto mutuo entre el docente y la persona mayor, entre la entidad académica y el estudiante. 
Sumado a lo anterior, se tiene en cuenta la concepción de Bru Ronda (2011), quien refiere la necesidad de profundizar en los análisis de las enseñanzas específicas para mayores en las universidades, entendidas como formación permanente y a lo largo de la vida, necesarias en una sociedad democrática e igualitaria, dispuesta a responder al reto del envejecimiento de la población, con vistas a consolidar una sociedad abierta a todas las edades, capaz de reducir la pobreza y fomentar la inclusión de grupos marginados y la participación social, además de promover una ciudadanía activa.

Según Meza y Villalobos (2008), en el proceso de envejecer se experimenta la constante de que la capacidad física decrece, mientras que algunos rasgos se incrementan, como la capacidad intelectual y la experiencia de la vida, entre otros. La sociedad actual sobrevalora la juventud, el trabajo productivo, el poder de compra; sin embargo, es una realidad que en el futuro habrá muchos adultos mayores, y la sociedad no se está preparando para ello. La última parte de la vida debería tomarse como una oportunidad de crecimiento, como la culminación de un proceso natural e inevitable, donde los educadores profesionales puedan visualizar en ese momento de la vida una oportunidad para intervenir educativamente y promover en el adulto, próximo a la jubilación, una actitud positiva frente a su transformación ocupacional, lo que conlleva un cambio en su modo de vivir, de pensar y de actuar ante el retiro del trabajo, con todo lo que ello implica.

Estos autores también refieren que las escuelas y universidades preparan para la vida activa, y las empresas se ocupan de la formación de sus empleados con miras a incrementar su productividad, pero ni la educación formal ni la no formal consideran una preparación que permita afrontar de manera positiva la vida después del trabajo, no obstante que la juventud terminará convirtiéndose en una minoría, toda vez que la pirámide poblacional se invierte peligrosamente. Que la persona aprenda una nueva relación con la sociedad, al llegar a la vejez, es tarea educativa. Y si se cree que una parte considerable de la vida transcurre en el trabajo, en torno al cual se organiza cada día (pues determina las horas de levantarse, de comer y de regresar a casa), es evidente que dejar de trabajar implica mucho más que el cese de la actividad productiva. Al llegar a la adultez tardía, dejar de realizar un trabajo productivo suele ser consecuencia de la jubilación, hasta el punto de que predominantemente se considera la tercera edad una época de crisis, pues en la etapa de jubilación la persona necesita readaptarse al medio, una vez que se encuentra fuera de la rutina laboral (Meza y Villalobos, 2008).

Teniendo en cuenta las razones descritas, en la región es factible que se ofrezcan programas de formación para la población adulta mayor, derivados de la política pública que se tenga en el momento o de organizaciones cuyo objetivo sea la atención del adulto mayor. Sin embargo, a pesar de la existencia de una red de instituciones educativas y universidades en la ciudad de San Juan de Pasto, no se tiene claridad al respecto. Quizás juegue allí el interés de establecer programas de educación permanente para adultos mayores partiendo de un modelo no fundamentado en sus intereses, destrezas y habilidades, independiente de su escolaridad, ni en las estrategias de enseñanza y aprendizaje derivadas de la Andragogía, con sus docentes universitarios e instituciones educativas para adultos. Ello abriría nuevos caminos para ofrecer servicios de educación y cambios de paradigmas que favorezcan la inclusión y participación de los adultos mayores como seres productivos y valiosos para la sociedad.

Al hablar de educación para adultos mayores se consideran los aportes de Yuni y Urbano (2008), quienes resaltan la aparición de la educación permanente, base de la educación de adultos mayores, como un proyecto para desarrollar todas las posibilidades de formación fuera del sistema educativo, lo que abarca las dimensiones de la vida, las ramas del saber y los conocimientos prácticos que puedan contribuir al desarrollo de la personalidad. Esto implica fines tales como: 
- Ofrecer oportunidades de exploración y potenciación de habilidades.

- Promover la adaptación ante las transformaciones sociales, culturales y valores, con uso de sus recursos personales, su motivación, sus intereses y su sentido de vida.

- Profundizar las razones de vivir, facilitando la integración social, con adecuada autoeficacia percibida.

- Generar en los adultos mayores mayor autoconfianza y seguridad, para que sean autónomos y tomen iniciativas.

Así, considerar como población de investigación a los trabajadores de la Universidad Mariana en proceso de prejubilación abre a esta la posibilidad de proyectarse socialmente, cumpliendo sus principios y valores. Y si bien las mujeres no alcanzan la edad de adulto mayor, pues su tiempo de jubilación es a los 57 años, como los hombres se jubilan a los 62 años (Ley 797 de 2003), de acuerdo con la Ley 1251 de 2008, sí están en el rango de adultos mayores. Hay que tener en cuenta, además, que la jubilación es un evento que modifica o transforma el rol laboral (Villar, 2013) y, por ende, todo el proceso de vida, ya que cambian las costumbres, las rutinas, y que son pocas las instituciones que toman en cuenta el periodo de preparación, es decir, la etapa de prejubilación, para proveer de mecanismos o estrategias que ayuden a afrontar dichos cambios desde una perspectiva personal, guiado por sus intereses, motivaciones y sentido de vida, para continuar su desarrollo personal.

La Universidad Mariana, en su visión de alcanzar la acreditación, propone en su plan de desarrollo -específicamente en lo que toca a la planeación estratégica de la Oficina de Gestión Humana- la meta de realizar los planes de retiro como parte del proceso de prejubilación de sus trabajadores. Así se incluiría la formación permanente a partir de los intereses, motivaciones y sentido de vida de los prejubilados, pues no existe proceso alguno en la historia de la Institución, y sería una manera novedosa, tanto para las investigadoras como para la Universidad, de desarrollar una investigación que dé cuenta y aporte en la construcción de un plan de formación permanente, inicialmente orientado a sus colaboradores.

Es importante, además, llevar a la práctica los postulados de la filosofía institucional, orientada a contribuir en la transformación social, con justicia social. Por tanto, esta propuesta es un medio para hacer operativos dichos postulados en el propio contexto, a través del plan de prejubilación, y una manera de fortalecer el sentido de vida, atendiendo a los propios intereses y motivaciones de los beneficiarios. Esto llevará a tener una actitud de merecimiento, a concretar objetivos y metas y a asumir retos frente al disfrute de la jubilación como una oportunidad de bienestar.

La investigación es innovadora, pues es la primera experiencia que se plantea con esta perspectiva, tanto en lo institucional como regionalmente; además, es pertinente para el proceso investigativo, ya que "el interrogante de la vida puede ser contestado si asumimos nuestra vida con responsabilidad, que es el sentido de nuestra existencia" (Frankl,1984, p. 250), existencia que en los adultos mayores puede ser productiva y exitosa cada día, en los diferentes contextos donde interactúan.

Además, el plan de prejubilación basado en los intereses, motivaciones y sentido de vida llevará a la persona prejubilada a ser más productiva en su desempeño, al visionar un futuro agradable, como retribución de un tiempo entregado a la institución, y a ser más "creativa cuando logra extraerle sentido a una vida que parecía absurda. La vida es potencialmente significativa hasta el último momento, hasta el último aliento" (Frankl, 1984, p. 246). Por esto, la propuesta es un gran aporte para los empleados y la institución, y cumple con la responsabilidad social institucional, en su propio contexto y realidad laboral.

Para el proyecto de investigación "Intereses de formación, motivación y sentido de vida de los 
trabajadores en proceso de prejubilación de la Universidad Mariana" se tuvo en cuenta el proceso de envejecimiento, que la mayoría de las veces genera una desestabilización personal. Ello ocurre por los cambios biológicos, psicológicos, sociales y culturales, así como en el sistema de valores de su contexto, donde se desconocen las razones de vivir y las formas de integración social que llevan a la desconfianza en sí mismo, inseguridad ante la toma de decisiones frente a los nuevos retos que tiene que asumir el adulto mayor y los compromisos que le faltan por cumplir, según sus expectativas e intereses.

Según el último censo del Departamento Administrativo Nacional de Estadística (DANE), en Colombia la población mayor de 60 años en el año 2011 era de $166.305-79.500$ hombres y 87.115 mujeres-, y para el 2020 se espera una población, en el mismo rango de edad, mayor a 6.435.899. Esto impone dificultades, no solo como consecuencia de un proceso de envejecimiento inadecuado, sino también por la baja cobertura que esta población tiene en el sistema de seguridad social, pensando en que se le garanticen adecuadas condiciones de vida. Sumando a esto, en el sistema pensional, en el momento de acercarse a su jubilación o cese laboral, los trabajadores enfrentan condiciones que afectan sus roles, hábitos, rutinas y participación social. En el ámbito regional, específicamente en la ciudad de Pasto, un estudio de Rosas, Paredes, Yarce, Rosero y Morales (2015) arroja factores de riesgo, como el deterioro cognitivo y la depresión, que deberían ser atendidos mediante una estrategia educativa, teniendo en cuenta que son factores protectores para el envejecimiento activo y exitoso (Yuni y Urbano, 2011).

Esto exige conocer y determinar los intereses de formación, motivación y sentido de vida de los trabajadores en proceso de prejubilación de la Universidad Mariana, con el fin de tener presentes las características y motivaciones de esta población, como línea de base para proponer un plan de retiro, que se construye en la planeación estratégica del proceso de Gestión Humana de la Universidad Mariana.

\section{Metodología}

La investigación científica permite llegar al conocimiento, con su descripción, explicación, interpretación y transformación de una realidad, donde el método científico proporciona los medios para el análisis crítico de la información obtenida de una población, situación, problemática o contexto especifico (Monje, 2011, p. 8). Es así que, para este estudio, se ha seleccionado un paradigma cuantitativo, de enfoque empírico analítico y descriptivo (Sampieri, Collado y Baptista, 2010), que permita identificar los intereses de formación permanente de un grupo de personas en proceso de prejubilación, como trabajadores de la Universidad Mariana.

La población estuvo conformada entonces por trabajadores en proceso de prejubilación, y la muestra, de tipo no probabilístico por conveniencia, la integraron mujeres mayores de 55 años y hombre mayores de 60 años, sin deterioro cognitivo, con comunicación oral y escrita, con deseo de participar voluntariamente (consentimiento informado) respondiendo la encuesta. El total de la muestra está conformado por 24 trabajadores.

Se utilizó como técnica la encuesta, la cual consiste en obtener información de los sujetos de estudio, en primera instancia, sobre aspectos sociodemográficos relacionados con edad, sexo, escolaridad, ocupación anterior y actual, ingreso económico y estado civil; en segundo lugar, sobre el potencial interés en temas de formación en salud, informática, artística, conocimientos y relaciones humanas, con respuestas tipo Likert, y, por último, sobre las razones por las cuales le gustaría estudiar. De igual manera, se obtuvo información cualitativa a través de preguntas abiertas, relacionadas con el sentido de vida, que aportaron un complemento importante para los resultados de los datos obtenidos. Se realizó la validación del Instrumento Exploración de Intereses de Formación para Adultos Mayores por prueba piloto.

Para el análisis de los datos obtenidos, se utilizó el Paquete estadístico SPSS, con el aporte de 
ISSN 0123-1294 | e-ISSN 2027-5358 | Educ.Educ. Vol. 21. No.1 | Enero-abril de 2018 | pp. 95-113.

Universidad de La Sabana | Facultad de Educación

estudios previos en el campo de la investigación, y para los datos cualitativos, tomados como apoyo del análisis de los datos, se realizó una matriz de vaciado de información.

\section{Resultados}

La población objeto de investigación, correspondiente a 24 trabajadores en proceso de prejubilación de la Universidad Mariana, se encontraba en un rango de edad de 55 a 59 años (41,6\%), con prevalencia del sexo femenino $(79,2 \%)$ y estrato socioeconómico 2 (37,5\%), 45,8\% casados. En escolaridad, $29,2 \%$ han realizado estudios de pregrado y $20 \%$, estudios de primaria. La población de estudio evidencia gran variedad de ocupaciones realizadas antes de trabajar en la Universidad Mariana, entre las cuales se destaca la ocupación como ama de casa (25\%), con una baja presencia de ocupaciones relacionadas con cargos como asistente o auxiliar administrativo.

Con respecto a la ocupación actual, la gran mayoría de los entrevistados se desempeñan en el cargo de servicios generales (33,3\%), seguidos del cargo de auxiliar administrativo (20,8\%), con una actitud favorable a la participación en procesos de formación $(87,5 \%)$, lo que es determinante para la viabilidad de la generación de procesos de formación permanente en la Universidad Mariana, orientado a esta población específica.

\section{Tabla 1. Motivaciones para participar} de un proceso de formación

\begin{tabular}{|l|c|c|}
\hline \multicolumn{1}{|c|}{ Razones } & No. & $\%$ \\
\hline Aprender algo nuevo & 13 & 54,2 \\
\hline Aprender a trabajar & 3 & 12,5 \\
\hline Compartir experiencias & 4 & 16,7 \\
\hline Hacer amigos & 2 & 8,3 \\
\hline Hacer voluntariado & 5 & 20,8 \\
\hline Ocupar su tiempo libre & 3 & 12,5 \\
\hline Recreación & 3 & 12,5 \\
\hline
\end{tabular}

Fuente: elaboración propia.
Al indagar acerca de las motivaciones por las cuales les gustaría participar en un proceso de formación, se observó que algunos de los trabajadores tenían más de una razón para hacerlo, siendo evidente que la razón de mayor porcentaje $(54,2 \%)$ es la de aprender algo nuevo, seguida de la razón de hacer voluntariado.

Tabla 2. Intereses por temas de formación

\begin{tabular}{|c|c|c|c|c|c|c|}
\hline Temas & Ninguno & $\%$ & Alguno & $\%$ & Muchos & $\%$ \\
\hline \multicolumn{7}{|c|}{ SALUD } \\
\hline Nutrición & 3 & 12,5 & 7 & 29,2 & 14 & 58,3 \\
\hline $\begin{array}{l}\text { Primeros } \\
\text { auxilios }\end{array}$ & 6 & 25,0 & 7 & 29,2 & 11 & 45,8 \\
\hline $\begin{array}{l}\text { Estilos de vida } \\
\text { saludable }\end{array}$ & 6 & 25,0 & 5 & 20,8 & 13 & 54,2 \\
\hline \multicolumn{7}{|c|}{ INFORMÁTICA } \\
\hline $\begin{array}{l}\text { Procesamiento } \\
\text { de datos }\end{array}$ & 5 & 20,8 & 7 & 29,2 & 12 & 50,0 \\
\hline $\begin{array}{l}\text { Acceso virtual / } \\
\text { e-learning }\end{array}$ & 7 & 29,2 & 5 & 20,8 & 12 & 50,0 \\
\hline \multicolumn{7}{|c|}{ ARTÍSTICA } \\
\hline Música & 10 & 41,7 & 9 & 37,5 & 5 & 20,8 \\
\hline Artes plásticas & 14 & 58,3 & 5 & 20,8 & 5 & 20,8 \\
\hline Historia del arte & 13 & 54,2 & 9 & 37,5 & 2 & 8,3 \\
\hline \multicolumn{7}{|c|}{ CONOCIMIENTOS } \\
\hline $\begin{array}{l}\text { Literatura y } \\
\text { filosofía }\end{array}$ & 12 & 50,0 & 5 & 20,8 & 7 & 29,2 \\
\hline $\begin{array}{l}\text { Agricultura } \\
\text { urbana }\end{array}$ & 12 & 50,0 & 5 & 20,8 & 7 & 29,2 \\
\hline Idiomas & 11 & 45,8 & 7 & 29,2 & 6 & 25,0 \\
\hline Emprendimiento & 8 & 33,3 & 4 & 16,7 & 12 & 50,0 \\
\hline \multicolumn{7}{|c|}{ RELACIONES HUMANAS } \\
\hline $\begin{array}{l}\text { Resolución de } \\
\text { conflictos y } \\
\text { liderazgo }\end{array}$ & 4 & 16,7 & 6 & 25,0 & 14 & 58,3 \\
\hline $\begin{array}{l}\text { Espiritualidad } \\
\text { franciscana }\end{array}$ & 6 & 25,0 & 2 & 8,3 & 16 & 66,7 \\
\hline $\begin{array}{l}\text { Manejo de } \\
\text { emociones y } \\
\text { sentimientos }\end{array}$ & 3 & 12,5 & 6 & 25,0 & 15 & 62,5 \\
\hline
\end{tabular}

Fuente: elaboración propia.

Al explorar los intereses de temas de formación, se evidencia que la gran mayoría hay mucho interés en salud, informática, relaciones humanas y emprendimiento. 
Tabla 3. Sentido de vida, expectativas y participación

\begin{tabular}{|l|c|c|c|c|}
\hline \multicolumn{1}{|c|}{ Pregunta } & Si & $\%$ & No & $\%$ \\
\hline $\begin{array}{l}\text { Encuentra sentido a la vida a través } \\
\text { del trabajo }\end{array}$ & 23 & 95,8 & 1 & 4,2 \\
\hline $\begin{array}{l}\text { Al jubilarse tiene la expectativa de } \\
\text { volver a trabajar }\end{array}$ & 18 & 75,0 & 6 & 25,0 \\
\hline $\begin{array}{l}\text { Al jubilarse le gustaría pertenecer a } \\
\text { una asociación }\end{array}$ & 16 & 66,7 & 8 & 33,3 \\
\hline
\end{tabular}

Fuente: elaboración propia.

Se evidencia que la mayoría de los trabajadores responden afirmativamente al gran sentido que le proporciona el trabajo a su vida, teniendo expectativas para continuar laborando y también interés por pertenecer o participar de una asociación.

Para dar mayor sustento a las preguntas anteriormente descritas, se aplicó una encuesta con preguntas abiertas, como: ¿Usted encuentra un sentido de vida a través de su trabajo? ¿Cuáles son sus emociones y sentimientos frente a la jubilación? ¿Al jubilarse tiene la expectativa de tener una nueva oportunidad de trabajo? ¿Al jubilarse le gustaría vincularse a una asociación, club o voluntariado? Los resultados obtenidos de esta encuesta justifican desarrollar un plan de retiro para los trabajadores en proceso de prejubilación, lo cual se ve reflejado en la siguiente discusión.

\section{Discusión}

Al reflexionar sobre el envejecimiento y la vejez, se piensa inicialmente en sus implicaciones corporales. Como ya se ha explicado, el envejecimiento se entiende como un proceso fundamental biológico con cambios físicos, mentales, individuales y colectivos, es decir, bioquímicos, fisiológicos, morfológicos, sociales, psicológicos y funcionales, que aparecen en el individuo a lo largo de la vida como consecuencia de la acción del tiempo (Arce y Ayala, 2012). Por su parte, con un enfoque demográfico, la vejez comprende una primera etapa, que corresponde a la prejubilación y la jubilación, cuando se tiene en promedio 60 años, y una etapa final, donde se pierde la independencia, después de los 80 años. Desde este punto de vista, en la actualidad es importante el enfoque demográfico, ya que la pirámide poblacional está variando y se incrementa la esperanza de vida (Ayuso y Holzmann, 2014).

Al determinar las implicaciones del envejecimiento, asimismo determinado por la normatividad atinente a la participación laboral, es importante tener en cuenta la formación que se dé en la prejubilación (Dornell, 2014) y un tiempo suficiente de adaptación al retiro, con base en unas prácticas y en los intereses y recursos que tienen tanto de la población como la institución. Tal y como lo expresa Dornell, los jubilados, "al pasar a la condición de no ocupados en el mundo del trabajo, se sienten abandonados de todo lo que ha motivado su dinámica habitual, y rodeados o abrumados de problemas que no esperaban y que a su vez no saben cómo afrontar" (2014, p. 3).

El interés en participar en procesos de formación, motivado por el deseo de querer aprender algo nuevo, ratifica que el sujeto envejece tiene las mismas o mayores posibilidades de seguir aprendiendo y replantear su proyecto de vida, con lo que se desmitifica la sobrevaloración del trabajo como proyecto de vida, al igual que el proceso de envejecimiento (Dornell, 2014), tomándolo como un proceso para optimizar oportunidades de participación, salud y seguridad que mejoren la calidad de vida (Zunzunegui y Béland, 2010). Estos resultados contrastan con los obtenidos en el estudio de Yuni y Urbano (2005), que igualmente indagó los motivos para participar en actividades educativas y obtuvo como mayor resultado el de deseo de mantenerse activo física e intelectualmente $y$, con un menor puntaje, de aprender cosas que sirvan para mejorar la situación personal o algún otro fin particular.

Al tener conocimiento de las características sociodemográficas de la población de trabajadores en proceso de prejubilación de la Universidad Mariana, es posible establecer los mecanismos o las estrategias 
apropiadas, pues de ellas dependen el desarrollo de las motivaciones y la presencia de un sentido de vida, tal y como lo expresa Chong (2012). Y las motivaciones también dependen de las ocupaciones ejercidas, las interacciones sociales, el proceso educativo y las habilidades sociales que se han tenido a lo largo de la vida y han permitido contactarse con una realidad y necesidades, en este caso, en un ámbito universitario. Con ello, además, se reafirman la creencia en la propia capacidad de adaptación y transformación en el ciclo vital de envejecimiento.

Dado que la mayoría de trabajadores son mujeres y en edades de 55 a 59 años, con algún nivel educativo, y teniendo en cuenta que en Colombia la mayoría de adultos mayores de 60 años son analfabetas (Zea, 2009) y, para el caso de los trabajadores de la Universidad Mariana, la gran mayoría poseen estudios mínimamente en primaria, aunque hay población con estudios secundarios y superiores, lo anterior representa una gran ventaja a la hora de ofrecer programas de formación acordes a sus necesidades y posibilidades. Por otra parte, como en su mayoría la población de la Universidad Mariana es femenina -dato acorde con la tasa de feminización demográfica mundial- hay mayor interés por la formación, en contraste con lo expresado por los hombres, quienes refieren ocupar su vida en familia. Esto coincide con lo expuesto por los Yuni y Urbano (2011), quienes manifiestan que los varones optan más por el ocio y la recreación, mientras que a las mujeres las motivada la búsqueda de nuevas experiencias personales y sociales, lo que les permiten integrarse y participar más fácilmente que los hombres, quienes permanecen relegados en casa, asumiendo nuevos roles domésticos.

Con respecto a los temas de mayor interés, los trabajadores en proceso de prejubilación están inclinados por temas de salud, informática, relaciones humanas y emprendimiento, dato de especial significación para las investigadoras, pues en la bibliografía que trata esta temática Yuni y Urbano (2011) encontraron diferencias en los tipos de cono- cimiento considerado, pues, por un lado, se interesan por conocimientos instrumentales, para trabajar y ganar dinero, y conocimientos para la vida, que se apropian a partir de la reflexión, el encuentro ínter e intrageneracional y la vivencia, frente a lo conocido académicamente, en el que además existe un tiempo diferente, que es productivo, útil y significativo y aquel tiempo existencial, en donde se llega a la autocomprensión, al fortalecimiento de la autoeficacia percibida, al encuentro de su sentido de vida. Estos conceptos hacen la diferencia, al plantear unas estrategias de formación como parte de un plan de retiro, pues no se deberán generar programas o cursos de formación permanente de la misma manera, como se hace en los programas de pregrado y quizás de posgrado, sino de manera que el tiempo y el conocimiento sean pautados por individuos que ya han vivido experiencias valiosas y tienen motivaciones diferentes para aprender o incursionar en el mundo académico, razón por la cual están implicados más valores personales y espirituales que aquellos que motivan a ganar dinero o mantener un nivel de vida, ya alcanzado en todo su trasegar laboral.

Según Kielhofner (2004), el ser humano presenta cambios necesarios a lo largo de su vida, que pueden no ser generados por sí mismo y requieren de factores externos, físicos, sociales o psicológicos para producirse. Estos cambios pueden traer consigo alteraciones en las rutinas, roles o propósitos de vida, más marcados en la época de la jubilación laboral, ya que el trabajo se convierte en el mediador de los horarios, las rutinas, los roles y las relaciones y, al perderse estos, las actitudes frente a la vida se alteran o se transforman. Cambios que pueden ser negativos, si desde mucho antes de la jubilación se consideraba el trabajo como único sentido de vida y si, al percibir este cambio, el individuo vive la paradoja de querer finalizar aquello que lo mantenía ocupado y lo cansaba, mientras que, por otra parte, ya lo extraña, por haber sido la pauta de su vida diaria.

Es entonces cuando se ve la importancia de conocer el sentido de vida, las emociones, sentimientos, 
expectativas y metas de los trabajadores en proceso de prejubilación, para poder proponer estrategias de formación en un plan de retiro efectivo, donde, además de la adquisición de nuevos conocimientos productivos, se posibilite la búsqueda de un nuevo sentido de vida, para llegar a un envejecimiento activo, exitoso y satisfactorio.

Cuando en nuestro contexto se habla de educación para adultos mayores, es visible el escepticismo frente a la posibilidad que tienen estos de seguir aprendiendo. Y si bien siempre estará vigente la máxima de Confucio "nunca es tarde para aprender", la educación para los mayores es poco valorada, aunque existen experiencias internacionales exitosas. Por ejemplo, en Taiwan se enseña temas de salud familiar, seguridad alimentaria, seguridad de transporte, para que los adultos sean mentores o transmisores en otras instituciones (Lee, 2015); en Finlandia se enseña computación o informática, con el objetivo de disminuir los niveles de soledad y deterioro cognitivo (Blažun, Saranto y Rissanen, 2012); en Chile, Brasil y Argentina, se instituyeron desde los años 80 las universidades del adulto mayor o de la tercera edad, que orientan la obtención de especializaciones para el ejercicio de roles sociales basados en el voluntariado (Yuni y Urbano, 2008a), y en España utilizan como estrategia la información y comunicación tecnológica para disminuir la brecha digital, además de desarrollar o mantener habilidades motoras y cognoscitivas para favorecer la inclusión (Guardiola, Vázquez y Salguero, 2012).

En Colombia, se está iniciando este proceso de formación universitaria de adultos mayores en la ciudad de Medellín (Hincapié, 2011), con una cátedra que incluye módulos de sistemas, literatura, mantenimiento cognitivo, historia y cultura, gerontología y gimnasia oriental. Se trata de cambios significativos en las actitudes frente a esta temática, soportados en la geragogía. Estas son algunas de las experiencias.

En la mayoría de estas experiencias encontradas a nivel internacional y nacional, se propende por que los profesionales y docentes a cargo de la educación de los adultos mayores conozcan las características del envejecimiento y los cambios determinantes en la vejez, de tal manera que se puedan establecer estrategias conducentes a una formación diferente, con un enfoque diferencial, amparado en el estado de derecho. Incluso las últimas investigaciones sobre educación para el adulto mayor dirigidas a los docentes estudian cómo deben ser los perfiles de educadores de mayores, las estrategias gerontagógicas y la visión de la universidad del adulto mayor (Marchena, 2014).

A continuación, se establece la relación de la categoría "Sentido de vida" con las subcategorías emergentes presentes en las respuestas de los trabajadores en proceso de prejubilación de la Universidad Mariana.

\section{Visión interna y externa del "Sentido de vida"}

La visión interna, como plantea Hernández (2002), tiene relación con la subjetividad individual y existencial, es decir, con lo más significativo para el ser humano. Asunto de orden endógeno, de por sí complejo, ya que está relacionado con el sentir y el ser y depende de la autoconciencia, "expresada en el impulso concienciado, adaptabilidad y toma de decisiones" (Lucero, 2016) y autogestión. La visión externa, por su parte, por ser exógena, se relacionada con las expectativas del entorno y depende de la gestión relacional, la "interacción y contagio emocional, [además de] experiencias familiares y sociales" y de la conciencia social, sujeta a la forma de observarse en el entorno (Lucero, 2016). Desde estas visiones vale indagar a los prejubilados con qué visión valoran la realidad y cómo expresan las experiencias, articulan las circunstancias y perciben las condiciones.

En este sentido, las tendencias al estancamiento cuando asumen la vejez, con solo pensar en la edad, y la pérdida de entusiasmo por la vida, los sentimientos de derrota y las actitudes de autocompasión, al pensar que ya no se es capaz de ofrecer nada a los demás, son asuntos que llevan a la pasividad y 
ISSN 0123-1294 | e-ISSN 2027-5358 | Educ.Educ. Vol. 21. No.1 | Enero-abril de 2018 | pp. 95-113.

Universidad de La Sabana | Facultad de Educación

a ensimismarse. Esto lo refuerza cierto sentimiento de insatisfacción, como se detecta en la siguiente declaración: "sin embargo, no todo está cumplido, no todos los proyectos se pueden realizar, esto me deja una sensación de descontento" [R13]. Este descontento hay que analizarlo, si se quiere fortalecer el sentido de vida antes de la jubilación, además porque puede conducir al estancamiento y dificultar la aceptación de la jubilación, si no se encuentra sentido para la vida, aparte de trabajar, y porque en este estadio las personas se tornan pasivas y negativas, al no identificar otras opciones que las haga sentir útiles.

En cambio, quien acepta la etapa evolutiva y disfruta de sus conquistas y logros, se convierte en alguien productivo, disfruta con amor y gratitud cada instante, alimentando positivamente su sentido de vida. Y esto ocurre al tomar conciencia de sus cambios cuantitativos en edad y cualitativos en madurez y se es menos egocéntrico que en etapas anteriores, se tiene una actitud proactiva y de adecuado funcionamiento, de acuerdo con las capacidades y oportunidades, y un equilibrio entre el trabajo, la recreación y los estilos de vida.

La vida, cuando se vive bien, se siente bien. En mi caso [puedo] estar seguro de haber nacido bien, $y$ esto permite a futuro tener más y mejores satisfacciones [R13]. Me siento realizada. Logré culminar todo lo que me propuse en muchos aspectos [R14]. A pesar de dificultades normales, mi vida nunca ha perdido el verdadero sentido [R16]. Porque mediante el trabajo, hacemos un quehacer significativo que afianza nuestra identidad y funciona como un espacio de desarrollo de nuestra existencia [R17].

Como se colige de los anteriores testimonios, el sentido de vida puede ser positivo, cosa que ayuda a mantener relaciones armoniosas con los demás, aceptando y preparándose para la jubilación, como etapa de proyección y disfrute.

\section{"Emociones y sentimientos" de dependencia e independencia laboral}

La capacidad de conquista y renuncia requiere del prejubilado autorregulación cognitiva y emocional, de modo que pueda "autogobernar y autodirigir las propias emociones y sentimientos, estados de ánimo, impulsos, percepciones y creencias, para el logro de propósitos en el desempeño personal y social" (Revelo, 2015) y desde alli proyectarse a la jubilación. Por ello fue necesario analizar si las emociones estaban ancladas a una vida laboral activa, porque, de ser así, pueden ser signo de estancamiento o de que para ellos el trabajo es el centro de la vida y su refugio y de que relegan lo demás a un segundo plano y, por tanto, de que no encuentren sentido por fuera del trabajo y permanezcan insatisfechos. En este caso, la jubilación se torna en un problema que los anula y en algunos casos los deprime.

Todo el tiempo lo he ocupado en mi trabajo. Considero que tengo sentimientos de tristeza [R2.2]. Tristeza por dejar de trabajar en la Universidad [R2.4]. Tristeza de irme de la institución [R2.6]. Yo, no experimento mucha emoción, ya que aqui en Colombia todavía tendría que trabajar muchísimo para hacerlo, ya que no viví mucho tiempo en el país y no creo poder hacerlo. Yo trabajo actualmente porque se me ha dado esa posibilidad de la que estoy agradecida [R2.8]. Hay momentos [en los] que pienso qué va a ser de mi vida después de la jubilación. Creo que es lo normal sentirse preocupado, si toda la vida se ha trabajado. Por lo general, siento nostalgia y desearía seguir ocupándome, no quedarme a esperar que llegue la vejez [R2.9].

Las anteriores verbalizaciones indican que existe una dependencia emocional de la vida laboral activa, lo cual dificulta la jubilación y, por tanto, requiere de un proceso de formación que favorezca la preparación para una nueva situación que, en vez de estancar a la persona, le genere sentido. Esto es aún más cierto, si la visión de la vida articula las siguientes circunstancias laborales: 
El trabajo me hace sentir vivo, el compartir con mis compañeros [R5]. La vida gira alrededor de la parte social de un individuo y una de esas partes se desarrolla en el trabajo [R8]. Es la razón para mantenerse vivo, activo, con salud, bienestar, en comunicación, en servicio [Rg]. El trabajo me permite seguir asumiendo con responsabilidad mis compromisos de vida consagrada [R14]. Todos los días comparto con mis estudiantes; el diario vivir de ellos y mío hace parte fundamental de mi vida [R1]. El trabajo es una necesidad vital. Espero poder permanecer ocupada en algo hasta que Dios lo disponga [Rg]. Me mantengo ocupada, me gusta mi trabajo [R1o]. Si no se trabaja no se hace nada, para la familia y sacar adelante a los hijos [R12]. Desde mi vocación, no tengo jubilación, y desde la misión hasta que muera [R2.11]. Por la edad ya no puedo trabajar [R3.4]. Para mí el trabajo es un medio para disfrutar la vida [R18]. Tristeza, miedo a determinadas situaciones nuevas, alegría, preocupación, depresión, felicidad y enojo algunas veces [R2.16]. Porque me gusta trabajar, no me gustaría quedarme sin hacer nada [R3.3].

Percepciones y valoraciones de la realidad del momento laboral, como si fuera la única opción de vida. Se prefiere depender de horarios y más tiempo pasar en el trabajo que en la casa. El trabajo es la forma de mantenerse ocupado y activo y la razón que dificulta emocionalmente la jubilación y causa una gran frustración, al no poder seguir laborando.

En cambio, cuando las emociones y sentimientos no están centrados en la vida laboral activa y se siente la satisfacción del deber cumplido y de que uno se merece el disfrute del tiempo, aparte del trabajo, entonces se tienen proyectos y se hacen planes para ocupar el tiempo en la recreación, en la formación, acordes a la edad y tiempo, armonizando y disfrutando de la familia, amistades y voluntariado.

Siento alegria, ya que considero que he trabajado lo suficiente [R2.3]. Soy consciente de que he trabajado y que tiene que venir gente joven. He entregado todo por la universidad y necesito irme a descansar. Me siento feliz, porque ya podría disfrutar el tiempo libre que me queda [R2.5]. Alegría porque pasará más tiempo con su familia [R2.4]. Alegría, felicidad, ya que saldré a descansar [R2.7, R7]. Alegría, un poco de incertidumbre, paz del deber cumplido [R2.10]. Feliz, emocionado, como a qué hora [R2.12]. Satisfacción frente a las metas realizadas [R2.14]. Es la oportunidad de hacernos cargo de nosotros mismos y poner en mi los propios valores, tomado como un descanso merecido, con tranquilidad, dedicando el tiempo a la familia y mí misma [R2.17]. Alegría, proyección para disfrutar, libertad en el manejo de tiempo y producción, esperanza de nuevas posibilidades para disfrutar la vida [R2.18]. Alegría. Me permite compartir más tiempo con mis seres queridos [R2.19]. Sí. Pero independiente, porque ya hay más gente preparada y joven [R3.5]. Me siento capaz de hacerlo y de asumir la jubilación [R3.10].

Como se ve en las anteriores respuestas, se trata de personas satisfechas que asumen la jubilación como la etapa de descanso que merecen y les corresponde y encuentra un sentido de vida en un quehacer independientemente de la labor que hoy realizan. Porque tienen una visión positiva de las condiciones tanto de la vida laboral como del momento de la jubilación, tal como se evidencia en las siguientes respuestas:

Me siento útil ante la familia y la universidad [R4]. Porque el aprendizaje en todos los aspectos es mutuo, porque estoy formando no solo excelentes profesionales, si no también mujeres y hombres de bien [RT]. Porque soy útily puedo pasar mi tiempo en el trabajo o en mi familia [R7]. La jubilación es el medio para lograr el cambio, dando oportunidades a otras personas más jóvenes [R8]. Puedo relacionarme con las demás personas y aportar en su formación [R19]. 
ISSN 0123-1294 | e-ISSN 2027-5358 | Educ.Educ. Vol. 21. No.1 | Enero-abril de 2018 | pp. 95-113.

Universidad de La Sabana | Facultad de Educación

\section{Expectativas y metas: dependencia laboral, disfrute o proyección}

En la teoría psicosocial de Erikson (2012), el ser humano soluciona sus conflictos de acuerdo con la etapa en que se encuentra. En el caso del prejubilado, este está en una etapa en que produce o se estanca, que es propicia para abordar una formación según sus intereses y motivaciones, y que pase a la etapa de la integridad con disfrute de la vida.

Mi sentido de vida, en muchos aspectos han tenido su valor y ellos permitirán fortalecer mis tiempos futuros, sin tener muy en cuenta los que no [R13]. El trabajo me permitió conocerme, [pues permite] descubrirse los talentos y capacidades y también [las] limitaciones, para superarlos [R17]. Ahora que me jubilo, me gusta dedicarme al hogar y considero que no me gustaría hacer parte de una asociación o club [R4.2]. No me gustaría un voluntariado, sino solo vivir cada día plenamente, sin afanes [R4.16].

Para la proyección y disfrute de la jubilación, hay que facilitar el proceso de un modo productivo, de allí que haya sido fundamental conocer las expectativas de los prejubilados, entre las cuales se destacan en forma positiva las siguientes:

Alegría al saber que voy a poder descansar [R2.2]. Saber que tendré tiempo para las cosas que antes no podía disfrutar enteramente. Mis sentimientos: tengo mucho para darle al mundo que me rodea, y mucho que aprender de los demás [R2.13]. Porque continúo en el ejercicio de mi profesión y de otras actividades afines con mayor libertad [R3.1]. Por mi edad y porque considero que debo descansar y quiero dedicarme al hogar. Me gustaría dedicarme a tejery bordar [R3.2]. Ya quiero descansary realizar otras actividades [R3.7, R3.8]. Si me retiro de la vida laboral activa, trataría de vincularme a otra actividad, así no sea remunerada, que me permita explorar otro campo, como es ayudar a otros [R3.13]. Hacer lo que siempre realicé parcialmente, para ejecutarlo en mi tiempo libre y suficiente. Mi meta es construir desde el campo y la naturaleza un mejor estar para las personas amantes de las orquideas, desarrollar un sitio para cultivarlas, intercambiarlas y generar otros ingresos [R3.14]. Seguir en el proceso de formación espiritual [R3.15]. Me gustaría aprender informática [R3.16]. Compartir más momentos con mi familia, actividades que mejoren mi estado fisico y mi salud. Viajar [R3.18]. Se quiere cumplir con metas que le permite disfrutar lo que sabe y quiere hacer y transmitir experiencias [R3.19] Puedo tener una actividad independiente [R4.3]. Me gusta estar y participar en la comunidad y trabajar [R4.5]. Un voluntariado me servirá para relacionarme y tener más conocimiento de todas esas asociaciones [R.4.6]. Sí. Es la realización después de pasar las metas básicas como madre, esposa, hija y trabajadora, me gustaría el área de servicios [R4.7]. Participar en un voluntariado para distraerme y pasar tiempo [R4. 8]. Sí. Porque asi una partecita del tiempo libre se pude dedicar a los demás y en su beneficio, por ejemplo, con niños o con ancianos que tienen una limitación [R4.9]. Sería emocionante poder experimentar una nueva ocupación. Seguramente le podría dar el sentido a seguir la vida. Lo tomaría como un paso a un sentido de vida diferente. Suena muy interesante [R4.10]. Me gustaría conocer nuevas personas, ayudar a otras personas, aprender cosas nuevas, conocer personas nuevas que estén en condición de jubilados. También para viajar, si es posible, conocer nuevos lugares [R4.12]. Sí. Se aprende cosas de los compañeros, se hace programas de salida, de recreación [R4.13]. Tendré más tiempo para dedicar a mi familia, mi esposa, mi madrey cultivar mi salud, mis relaciones familiares, dedicarle mejor tiempo a la espiritual y también a la recreación y vivir en el campo [R4.15]. Me gustaría estudiar, estar en paz conmigo misma, con la naturaleza [R4.17]. Si le gustaría participar de un voluntariado porque el dinamismo siempre será 
parte de mantener el espiritu de cada ser [R4.18]. Formar un voluntariado para ayudar a generar una red o grupos de apoyo para investigación, recreación, trabajo, producción intelectual, que facilite continuar articulada de alguna manera a la universidad [R4.19]. Crear un voluntariado para ayudar a las personas que más necesitan y comunidades. Asociación para la promoción y prevención a la tercera edad [R3.2O].

Como se puede analizar en esta investigación, el sentido de vida frente a la jubilación se puede fortalecer con base en las metas y proyecciones, a través de un plan de formación que dé respuestas a estas expectativas, para que cuando se jubilen tenga un horizonte creado acorde a sus necesidades y expectativas. Esto también vale para los casos que perciben esta etapa como un estancamiento, para que puedan lograr una mejor expectativa de la jubilación y la puedan disfrutar.

Según Morón y Cruz, la educación de adultos mayores se debe concebir como el aprendizaje a lo largo de la vida, concepto que fue promovido por la Unesco en 1995, quienes citan que este aprendizaje es "toda actuación formativa que tiende a facilitar conocimientos y destrezas a las personas en edad postescolar, así como a promover en ellas actitudes $y$ comportamientos valiosos, orientados a propiciar su perfeccionamiento personal y profesional y a la participación social" (2012, citados por Marchena, 2014, p. 111). A partir de este concepto de aprendizaje a lo largo de la vida, se establecen tres principios que deben enfocar el proceso de formación permanente: 1) la educabilidad de la persona en cualquier etapa de su vida; 2) poseer unidad y globalidad, es decir, en todos los niveles y modalidades educativas; y 3 ) de manera universal, respetar el derecho a la educación, en cualquier tiempo y lugar y con cualquier finalidad.

En consecuencia, se deben proponer estrategias basadas en metodologías activas, prácticas, reflexivas e interactivas, tal y como lo recomienda el Instituto de Mayores y Servicios Sociales (IMSERSO, 2011, pp. 295-296), que propone una metodología cooperativa constructivista, en la cual se estimulan procesos de reconstrucción, se proporcionan apoyos eficaces para potenciar roles que generen autonomía de las personas mayores y se utilizan didácticas de carácter psicocéntrico, con investigación compartida, espacios intergeneracionales, aprendizaje autónomo con una organización y utilización de los propios recursos, con un aprendizaje basado en estudios de caso reales y de su interés, y por último, desarrollando proyectos con una metodología científica.

Otras recomendaciones para la formación permanente de los adultos mayores establecen que la educación a lo largo de la vida es vital para el envejecimiento activo y que es fundamental alentar las relaciones intergeneracionales para divulgar las experiencias, la lectura, la cultura, las costumbres y los saberes, además de incluir los programas de preparación a la jubilación activa que promuevan el cambio de rol de envejecimiento pasivo por el activo, al culminar su periodo laboral e iniciar el cese de actividades laborales. Por último, pero no menos importante, es preciso que esa formación trascienda a la población desde infantes hasta adultos jóvenes y genere conocimiento acerca de los procesos de envejecimiento activo, teniendo en cuenta que ellos serán en un futuro la población que envejece y está envejecida (Junta de Andalucía, 2010, pp. 434-435).

\section{Conclusiones}

La determinación de intereses de formación, motivación y sentido de vida facilita identificar el deseo de aprender algo nuevo, lo cual desmitifica el proceso de envejecimiento y la sobrevaloración del trabajo como proyecto de vida. La formación orientada a trabajadores en proceso de prejubilación requiere un tiempo para la adaptación al retiro, mediante prácticas participativas, siempre resultado de los intereses, motivaciones, capacidades y recursos que poseen tanto los prejubilados como la institución. 
El tiempo y conocimiento que exigen los programas de formación en el plan de retiro deben ser acordados con la población en prejubilación, mediante el intercambio de las experiencias significativas que han vivido, las cuales fortalecen el deseo de aprender o incursionar en el mundo académico, más otras posibilidades que les permitan identificar sus valores personales y espirituales, que les ayudaran a mantener un nivel de vida saludable.

Para llegar a un envejecimiento activo, exitoso y satisfactorio, se requiere fortalecer el sentido de vida con nuevos elementos y otras posibilidades, así como de la adquisición de nuevos conocimientos productivos, donde las estrategias de formación que promuevan un plan de retiro eficiente son de vital importancia, para que se lleve a cabo un retiro emocionalmente sano.

El sentido de vida y trabajo se valora de acuerdo con la propia realidad y experiencias, que están articuladas a diferentes circunstancias y percepciones de las condiciones, las cuales, a su vez, determinan emociones y sentimientos que se manifiestan en la dependencia o independencia laboral, proceso que requiere ser identificado durante la preparación para la jubilación, fortaleciendo expectativas y metas que ayuden a alimentar el sentido de vida del prejubilado.

Para desarrollar estrategias que promuevan la formación permanente de los adultos mayores, en este caso de los trabajadores en proceso de prejubilación de la Universidad Mariana, es importante tener en cuenta unos principios básicos que pueden establecer la dinámica educativa, por ser una estrategia nueva, comprometida con el desarrollo humano y la formación para el trabajo, según lo dispuesto por el Ministerio de Educación Nacional. De acuerdo con Marchena (2014), al implementar la formación permanente, es necesario:

- Generar modelos de valores sociales, criterios de selección de materiales y estrategias gerontológicas apropiadas al contexto.

- $\quad$ Promover el uso de las TIC como herramientas facilitadoras de participación social de los adultos mayores.

- Sensibilizar y culturizar a la sociedad sobre la importancia del cuidado de la salud para obtener un envejecimiento activo.

- $\quad$ Proponer espacios de intercambio de saberes intergeneracionales para trabajar conjuntamente la temática del adulto mayor.

- Desarrollar investigaciones para la generación de modelos, estrategias y didácticas basados en la geragogía, apropiada para el adulto mayor.

- Formar profesionales idóneos para la formación de los adultos mayores.

\section{Referencias}

Arce, I. A. y Ayala, A. (2012). Fisiología del envejecimiento. Revista de Actualización Clínica Investiga, 17, 813.

Ayuso, M. y Holzmann, R. (2014). Longevidad: un breve análisis global y actuarial. Madrid: BBVA (mimeo).

Blažun, H., Saranto, K. y Rissanen, S. (2012). Impact of computer training courses on reduction of loneliness of older people in Finland and Slovenia. Computers in Human Behavior, 28(4), 1202-1212. 
Bru Ronda, C. (2011). Aprendizaje a lo largo de la vida, envejecimiento activo y cooperación internacional en los programas universitarios para mayores. IV Congreso Iberoamericano de Universidades para Mayores CIUUmM, Alicante, 27 al 30 de junio de 2011.

Chong, A. (2012). Aspectos biopsicosociales que inciden en la salud del adulto mayor. Revista Cubana de Medicina General Integral, 28(2), 79-86.

Colombia. Ministerio de Salud. Resolución número 8430 de 1993. Por la cual se establecen las normas científcas, técnicas y administrativas para la investigación en salud. Recuperado de http://www.minsalud.gov. co/Normatividad/RESOLUCION\%208430\%20DE\%201993.pdf

Congreso de la República de Colombia (2003). Ley 797 de 2003. Por la cual se reforma algunas disposiciones del sistema general de pensiones. Diario Oficial,45(079) (29 de enero). Recuperado de http://www.secretariasenado.gov.co/senado/basedoc/ley_0797_2003.html

Congreso de la República de Colombia. (2008). Ley 1251 de 2008. Por la cual se dictan normas para la protección, promoción y defensa de los derechos de los adultos mayores. Diario Oficial, 47(186) (27 de noviembre). Recuperado de http://www.alcaldiabogota.gov.co/sisjur/normas/Norma1.jsp?i=33964

Departamento Nacional de Estadística - DANE. Proyecciones anuales de población por sexo, según grupos quinquenales de edad 1985-2015. Recuperado de http://www.dane.gov.co

Dornell, T. (2014). Las cartografías corporales en la vejez y el envejecimiento: dispositivo de análisis en la prejubilación. In IX Jornadas de investigación, docencia, extensión y ejercicio profesional: "Transformaciones sociales, políticas públicas y conflictos emergentes en la sociedad argentina contemporánea", La Plata, octubre de 2014.

Erikson, E. H. (2012). El ciclo vital completado: Edición revisada y ampliada. España: Planeta.

Frankl, V. (1984). Hombre doliente. Madrid: Rialp.

Galvanovskis, A.y Villar, E. (2000). Revisión de vida y su relación con el autoconcepto y la depresión en el periodo de jubilación. Geriátrika: Revista Iberoamericana de Geriatría y Gerontología, 16(10), 40-47.

Guardiola, D. P., Vázquez, M. M. y Salguero, R. M. (2012). El caso de “El fogón de la experiencia”: Universidad de Huelva. Tecnologías de la Información y la Comunicación en Mayores. Aularia: Revista Digital de Comunicación, 1(2), 231-236.

Hernández, O. D’Ángelo (2002). Sentido de vida, sociedad y proyectos de vida. En Ética y sociedad (pp. 1-42). La Habana: Félis Varela.

Hincapié, N. (2011). Programa Cátedra Universitaria para Adultos Mayores en el Tecnológico de Antioquia. Senderos Pedagógicos 1(2) (julio-diciembre), 39-48.

IMSERSO - Instituto de Mayores y Servicios Sociales (2011). Libro blanco sobre el envejecimiento activo. Madrid: IMSERSO - Ministerios de Sanidad, Política Social e Igualdad. 
ISSN 0123-1294 | e-ISSN 2027-5358 | Educ.Educ. Vol. 21. No. 1 | Enero-abril de 2018 | pp. 95-113.

Universidad de La Sabana | Facultad de Educación

Junta de Andalucía (2010). Libro blanco del envejecimiento activo. Sevilla: Consejería para la Igualdad y Bienestar Social.

Kielhofner, G. (2004). Terapia ocupacional: modelo de ocupación humana:teoría y aplicación. Médica Panamericana.

Lee, Y. H. (2015). Older adult education: new public pedagogy in $21^{\text {st }}$ century Taiwan. Australian Journal of Adult Learning, 55(3), 460.

López, B. (2013). Empoderamiento y adultos mayores. Impacto de la participación de un grupo de adultos mayores en un programa educativo. En V. Montes de Oca (coord.), Envejecimiento en América Latina y el Caribe: enfoques en investigación y docencia de la Red Latinoamericana de Investigación en Envejecimiento (LARNA). México: Instituto de Investigaciones Sociales, Universidad Nacional Autónoma de México.

Lucero, S. (2016). Influencia de la autorregulaciòn cognitivo emocional en el desarrollo de competencias socioemocionales en la formaciòn inicial de docentes del Sur Occidente Colombiano. Pasto: Universidad Mariana.

Marchena, J. A. M. (2014). Educación y personas mayores. Revista Electrónica Interuniversitaria de Formación del Profesorado, 17(1), 121.

Meza, M. y Villalobos, E. (2008). La crisis de la jubilación como una oportunidad educativa. Educación y Educadores, 11(2) (diciembre), 179-190

Monje, C. A. (2011). Metodología de la investigación cuantitativa. Guía didáctica. Escuela Sur Colombiana, Facultad de Ciencias Sociales y Humanas. Programa de comunicación y periodismo, Neiva. Recuperado de http://carmonje.wikispaces.com/file/view/Monje+Carlos+Arturo+-+Gu\%C3\%ADa+did\%C3\%A1ctica+Me todolog\%C3\%ADa+de+la+investigaci\%C3\%B3n.pdf

Revelo, S. E. L. (2015). La autorregulación cognitivo-emocional. Una estrategia para el desarrollo de competencias socioemocionales. Unimar, 33(2).

Rosas, G., Paredes, Y., Yarce, E., Rosero, M. y Morales, A. (2015). Caracterización de los factores multidimensionales de las personas mayores de 60 años de la ciudad de San Juan de Pasto. Colección Resultado de Investigación. Pasto: Unimar.

Rowe, J. y Kahn, R. (1987). Human Aging: Usual and Successful. Science, 237, 143-149.

Rowe, J. y Kahn, R. (1998). Successful aging. Nueva York: Pantheon.

Sampieri, R., Fernández, C.y Baptista, P. (2010). Metodología de la investigación. 5 ed. Lima: McGraw-Hill.

Torres, A. (2009). La educación para el empoderamiento y sus desafíos. Sapiens, 10(1), 89-108.

Villar, F. (2013). Desafíos de la investigación interdisciplinaria en gerontología. Envejecimiento en América Latina y el Caribe: enfoques en investigación y docencia de la Red Latinoamericana de Investigación en Envejecimiento (LARNA). México: Instituto de Investigaciones Sociales, Universidad Nacional Autónoma de México. 
Yuni, J.y Urbano, C. (2005). Educación de adultos mayores. Teoría, investigación e intervenciones. 1 ed. Córdoba: Brujas.

Yuni, J. y Urbano, C. (2008). Condiciones y capacidades de los educadores de adultos mayores: la visión de los participantes. Revista Argentina de Sociología, 6(10), 184-198.

Yuni, J. y Urbano, C. (2008a). Cartografía de experiencias educativas con personas mayores en el ámbito latinoamericano. Revista Palabras Mayores, Un espacio de conocimiento e información sobre el Adulto Mayor, (1).

Yuni J. y Urbano C. (2011). Aprendizaje, envejecimiento y vejez: Notas fenomenológicas sobre derroteros de transformaciones subjetivas. En J. Yuni (comp.), La vejez en el curso de la vida. Argentina: Encuentro-UNCA.

Zea, M. C. (2009). La experiencia del aula universitaria de mayores: enseñanza-aprendizaje de cuidado y autocuidado. Investigación y Educación en Enfermería, 27(2), 244-253.

Zunzunegui, M. V. y Béland, F. (2010). Políticas intersectoriales para abordar el reto del envejecimiento activo. Informe SESPAS 2010. Gaceta Sanitaria, 24, 68-73. 